\title{
On the possibility of identifying heavy ion acceleration processes in the magnetotail of Mars
}

\author{
G. A. Kotova ${ }^{1}$, M. I. Verigin ${ }^{1}$, A. P. Remizov ${ }^{1}$, H. Rosenbauer ${ }^{2}$, S. Livi $^{2}$, W. Riedler ${ }^{3}$, K. Schwingenschuh ${ }^{3}$, \\ M. Tatrallyay ${ }^{4}$, K. Szegö ${ }^{4}$, and I. Apáthy ${ }^{5}$ \\ ${ }^{1}$ Space Research Institute of Russian Academy of Sciences, Profsoyuznaya 84/32, Moscow 117810, Russia \\ ${ }^{2}$ Max Planck Institut fuer Aeronomie, Postfach 20, 37189, Katlenburg-Lindau, FRG \\ ${ }^{3}$ Space Research Institute of the Austrian Academy of Sciences (IWF), Inffeldgasse 12, A-8010 Graz, Austria \\ ${ }^{4}$ KFKI Research Institute for Particle and Nuclear Physics, 1525 Budapest P.O. Box 49, Hungary \\ ${ }^{5}$ KFKI Atomic Energy Research Institute, 1525 Budapest P.O. Box 49, Hungary
}

(Received February 10, 2000; Revised June 29, 2000; Accepted July 21, 2000)

\begin{abstract}
The dependence of the energy of ions on their mass could be a useful tool for the identification of possible acceleration processes in the Martian plasma sheet. The study is based on a few cases when heavier ions and protons were registered simultaneously in the plasma sheet by the TAUS energy spectrometer, and on published data of the ASPERA instrument. The analysis of mass dependence of the ion energy in the plasma sheet suggests that frictional preacceleration of ions probably in the pole regions of the Martian magnetosphere can be responsible for not more than $40 \%$ of the energy gain of heavy ions in the plasma sheet, the cross-tail current sheet acceleration yields up to $70 \%$, and from 30 to $70 \%$ of the heavy ion energy is provided by the acceleration due to magnetic field line stresses (and/or electric field aligned acceleration).
\end{abstract}

\section{Introduction}

Plasma experiments TAUS (Rosenbauer et al., 1989) and ASPERA (Lundin et al., 1989) on board the Phobos 2 orbiter revealed that the Martian magnetosphere is dominated by ions of planetary origin, primarily by oxygen ions. The most intense fluxes of tailward streaming heavy ions (ions with mass/charge ratio $M_{i} / q_{i}>3$ ) were observed in the magnetotail plasma sheet (Rosenbauer et al., 1989; Verigin et al., 1991). Velocity and intensity of heavy ion fluxes reach their peak values in the vicinity of the magnetic neutral line. The energy of these ions varies from $\sim 100 \mathrm{eV}$ up to $>6$ $\mathrm{keV}$. 2D ion spectra measured by the TAUS spectrometer in the Phobos 2 elliptical orbits revealed a supersonic, highly anisotropic distribution function of heavy ions (Rosenbauer et al., 1989). Analysis of these 2D spectra at times when the spacecraft was spinning, suggests that the 3D distribution function of heavy ions has a "mushroom cap" shape similar to the shape of proton distributions in the plasma sheet boundary layer of the terrestrial magnetotail (Kotova et al., 1997).

Several acceleration mechanisms were invoked for explanation of such ion distributions in the Earth's magnetotail (Eastman et al., 1986). Simple field aligned acceleration with adiabatic deformation of distribution function was examined by Kotova et al. (1997) and it was demonstrated that this mechanism can account for the Martian magnetotail observations only in a case of initial preacceleration of ions

Copy right (C) The Society of Geomagnetism and Earth, Planetary and Space Sciences (SGEPSS); The Seismological Society of Japan; The Volcanological Society of Japan; The Geodetic Society of Japan; The Japanese Society for Planetary Sciences. to velocities of several tens of $\mathrm{km} / \mathrm{s}$. This preacceleration might occur by the direct interaction of ionospheric ions with magnetosheath plasma in the 'pole' regions of the Martian magnetosphere (Verigin et al., 1991). Ip (1992) considered ion acceleration due to the formation of neutral lines in the near-tail region of the Martian magnetosphere. Dubinin et al. (1993) showed that acceleration due to the magnetic field stress could explain an increase of ion energy toward the center of the tail. The effect of the magnetic field line stress can be expressed via the effect of the electric field raised by the charge separation (Haerendel, 1987).

On the basis of the TAUS ion spectrometer and MAGMA magnetometer data correlations between several plasma sheet and solar wind parameters were found which indicate importance of the magnetic field line stress acceleration and the central current sheet acceleration of heavy ions (Kotova et al., 1997, 2000). Meanwhile none of the proposed acceleration processes can explain all the features of the Martian plasma sheet.

In this paper the dependence of heavy ion energy on the ion mass will be examined on the basis of Phobos 2 plasma experiments. This dependence provides an additional useful tool for the identification of possible acceleration processes.

\section{Observations}

The TAUS ion spectrometer on board the Phobos 2 Mars orbiter measured fluxes of protons and heavy ions (mass/ charge ratio $M_{i} / q_{i}>3$ ) separately in the energy range of $0.03-6 \mathrm{keV}$. It provided data from 5 elliptical and $\sim 60 \mathrm{cir}-$ cular orbits. In circular orbits a 1D heavy ion spectrum was measured during $8 \mathrm{~s}$ in every $2 \mathrm{~min}$. Published data of the 
March 11, $19890100-0300$ UT

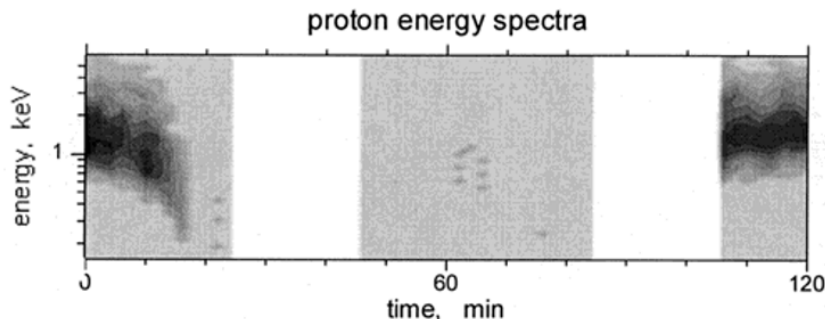

$\lg (\mathrm{cts})$
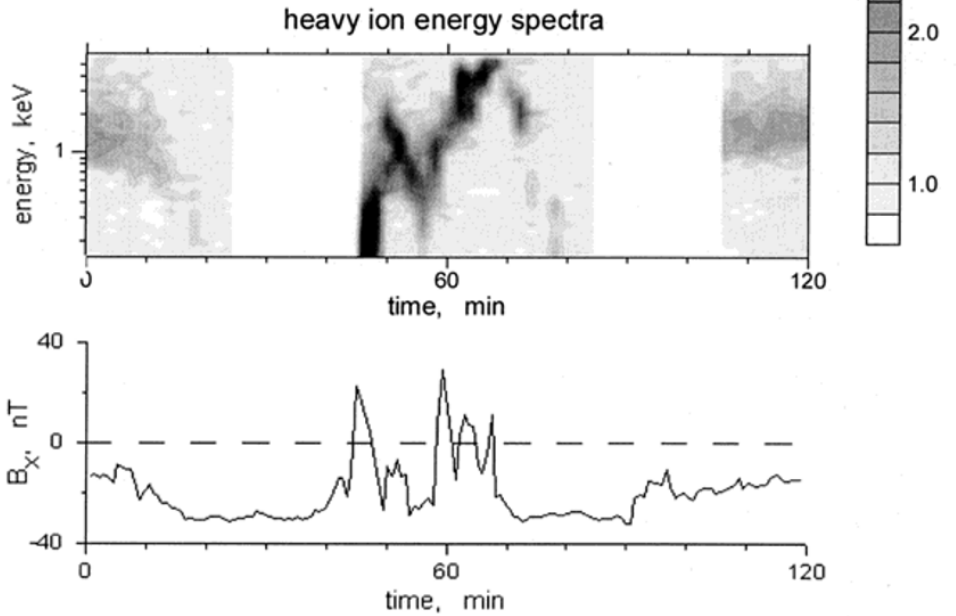

Fig. 1. Energy vs. time plot of proton and heavy ion spectra (in counts) and the magnetic field component parallel to Sun-Mars line, measured on March 11, 1989 inside the Martian magnetosphere.
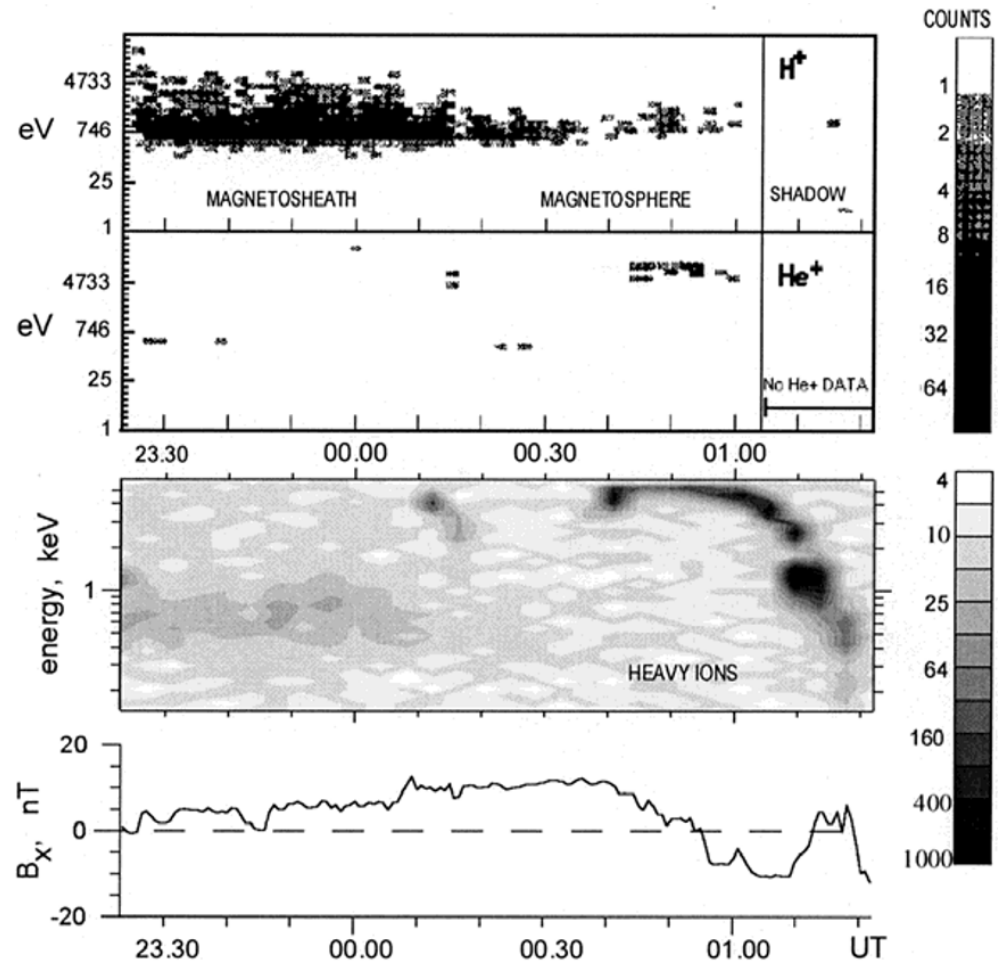

Fig. 2. Energy vs. time plot of proton and helium ion spectra obtained by the ASPERA instrument (top two panels) in the Martian plasma sheet on March 1-2, 1989 (Barabash et al.,1995) and corresponding heavy ion spectra measured by TAUS. Bottom panel presents the magnetic field $B_{x}$ component as observed by the MAGMA magnetometer. 
ASPERA ion mass-spectrometer (Lundin et al., 1989) will be also considered. This instrument included 10 moment sensors with a field of view of $5^{\circ}-36^{\circ}$ and measured positive ions in the range of $1-23 \mathrm{keV} / \mathrm{q}$. The ASPERA instrument also included mass-sensors which provided more detailed ion composition measurements but with lower sensitivity and time resolution compared to the moment sensors (Barabash et al., 1995).

Figure 1 presents example of proton and heavy ion measurements by TAUS when Phobos 2 passed through the Martian magnetotail on March 11, 1989. The bottom panel shows the magnetic field component parallel to the Mars-Sun line measured by the MAGMA magnetometer to visualize the magnetic neutral sheet traversals inside the magnetotail where this component changes sign. Rather high fluxes of protons (and 'ghosts' in the heavy ion channel) in the beginning and in the end of the presented time interval refer to the magnetosheath. Martian plasma sheet crossings are well seen in Fig. 1: close to multiple crossings of the magnetic neutral sheet the TAUS instrument recorded heavy ions and their energy varies through the entire instrumental energy range. In this case also protons were observed in the plasma sheet, while most of the time the sensitivity of TAUS was not enough for proton observations inside the tail. On March 11, 1989 the energy of protons in the plasma sheet was $\sim 4$ times less than the energy of simultaneously observed heavy ions.

More data on ion composition of the Martian plasma sheet were obtained by the ASPERA instrument due to its better mass resolution. Norberg et al. (1993) and Barabash and Norberg (1994) mentioned observations of helium ions $\mathrm{He}^{+}$ whose energy seems to be $\sim 1.5$ times less than the energy of oxygen ions. Figure 2 shows the results of hydrogen and helium ion observations by ASPERA (Barabash et al., 1995) and the data obtained by the heavy ion channel of TAUS. At the time close to the first current sheet crossing (0055 UT) heavy ions (supposedly oxygen) with peak energies higher than the TAUS instrument range were observed. ASPERA recorded rather high fluxes of protons and visible fluxes of helium ions at $\sim 0050 \mathrm{UT}$. Similarly to the first example the energy of protons $(\sim 1500 \mathrm{eV})$ is $\sim 4$ times less than the energy of oxygen ions, and the energy of helium ions (5-10 $\mathrm{keV}$ ) is close to the energy of oxygen or slightly less. Contrary to these observations Lichtenegger and Dubinin (1998) presented an example of $E_{i} / q_{i}-M_{i} / q_{i}$ matrix measured by the mass-sensor of the ASPERA instrument in the Martian tail, which shows approximately linear dependence of the energy $E_{i}$ on mass $M_{i}$ for light $\left(\mathrm{H}^{+}, \mathrm{H}_{2}^{+} / \mathrm{He}^{++}, \mathrm{He}^{+}\right)$and heavy $\left(\mathrm{O}^{+}, \mathrm{O}_{2}^{+}, \mathrm{CO}_{2}^{+}\right)$ions. However the matrix in figure 5 of Lichtenegger and Dubinin (1998) was collected during a long time interval of 35 minutes (March 16, 1021-1056 UT). In the same orbit but within shorter time interval (1029-1037 UT) enclosing the current sheet crossing ASPERA practically did not observe light ions (figure 2 in Norberg et al., 1993). Hence these ions might be not of plasma sheet origin but admixed, e.g., from the magnetospheric boundary layer.

The ASPERA data (Norberg et al., 1993; Dubinin et al., 1993 , etc.) revealed also that all heavy ions $\mathrm{O}^{+}, \mathrm{O}_{2}^{+}, \mathrm{CO}_{2}^{+}$ have nearly the same energy. Figure 3 taken from Dubinin et al. (1993) presents the peak energy of protons and molecular ions observed in the plasma sheet as a function of the peak energy of oxygen ions (solid and dashed lines will be discussed below). It is seen that the energy of protons is $\sim 2-$ 4 times less than the energy of oxygen ions, in agreement with TAUS observations. On the other hand the energy of molecular ions is approximately equal to the energy of $\mathrm{O}^{+}$. Using the ASPERA data, Dubinin et al. (1993) sometimes succeeded in identifying $\mathrm{O}^{++}$ions whose energy/charge ratio $E_{i} / q_{i}$ coincide with that of $\mathrm{O}^{+}$and concluded that $E_{i} / q_{i}$ of heavy ions does not depend on $M_{i} / q_{i}$.

The observations reported so far can be summarized as follows:

- energy of molecular ions $\left(\mathrm{O}_{2}^{+}, \mathrm{CO}_{2}^{+}\right)$is close to energy of $\mathrm{O}^{+}$ions;

- energy/charge ratio of $\mathrm{O}^{++}$is close to energy of singly charged $\mathrm{O}^{+}$ions;

- energy of protons is usually 2-4 times less than energy of $\mathrm{O}^{+}$ions;

- the energy of singly ionized helium seems to be $\sim 1.5$ times less than the energy of $\mathrm{O}^{+}$ions.

\section{Data Analysis and Discussion}

The acceleration processes which have been considered to explain the observed heavy ion fluxes are as follows:

- frictional preacceleration of all ions to velocity $V_{0}$;

- field-aligned electric field acceleration or magnetic field line stress acceleration of all ions to equal energy per charge $E_{i} / q_{i}=U$;

- cross-tail electric field acceleration of all ions which results in an approximately equal velocity increase $\Delta V=$ $2 c \varepsilon / B_{\perp}$, where $c$ is the velocity of light, $\varepsilon$ is cross-tail electric field, $B_{\perp}$ is magnetic field component perpendicular to the current sheet (Speiser, 1965; Shabanskiy, 1972). The energy gain of ions in this case is $\Delta E_{i}=$ $M_{i}(\Delta V)^{2} / 2+M_{i} V(\Delta V)$, where $V$ is the initial velocity of the ion.

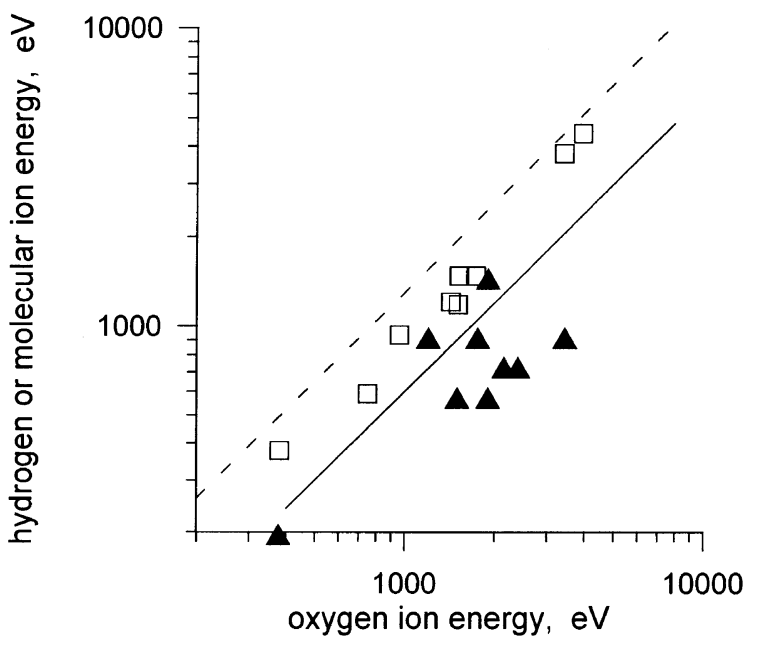

Fig. 3. Peak energy of protons (solid triangles) and molecular ions (open squares) versus peak energy of oxygen ions as measured by the ASPERA instrument (Dubinin et al., 1993). Solid and dashed lines show the relations: $E_{\mathrm{H}^{+}}=0.6 E_{\mathrm{O}^{+}}$and $E_{\mathrm{O}_{2}^{+}}=1.3 E_{\mathrm{O}^{+}}$, respectively. 


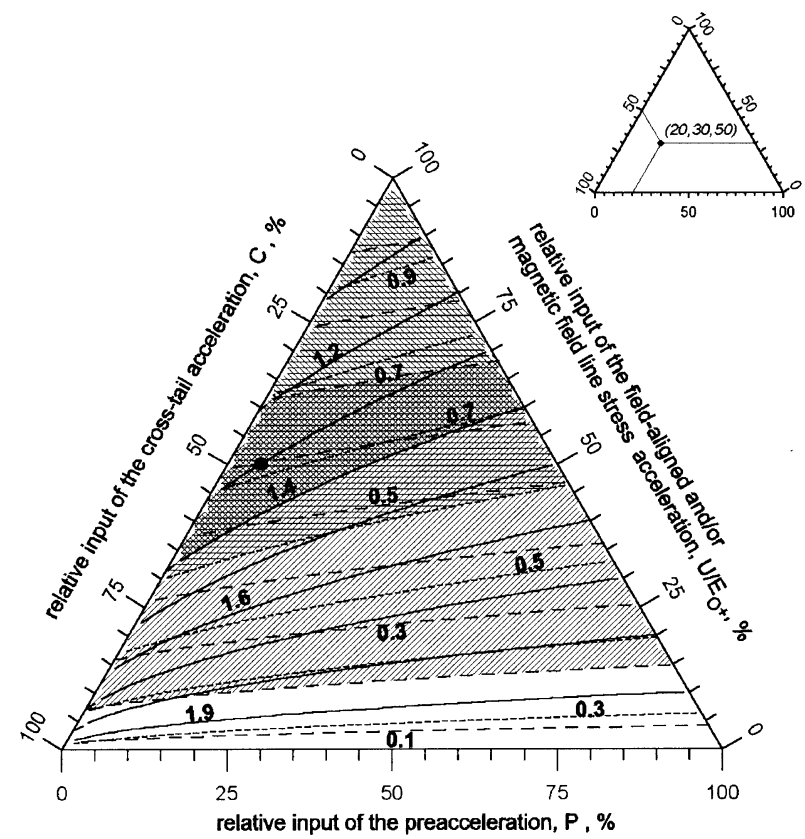

Fig. 4. Liquidus projection of isolines of $E_{\mathrm{H}^{+}} / E_{\mathrm{O}^{+}}$(dashed), $E_{\mathrm{He}^{+}} / E_{\mathrm{O}^{+}}$(dotted), and $E_{\mathrm{O}_{2}^{+}} / E_{\mathrm{O}^{+}}$(solid) in coordinates: energy gained due to preacceleration relative to the total energy vs. energy gained due to cross-tail current sheet acceleration relative to the total energy vs. energy gained due to field-aligned acceleration relative to the total energy for oxygen ions in the plasma sheet. Left hatching marks the region of $E_{\mathrm{O}_{2}^{+}} / E_{\mathrm{O}^{+}}<1.4$, right hatching shows the region of $0.2<E_{\mathrm{H}^{+}} / E_{\mathrm{O}^{+}}<0.7$ and horizontal hatching corresponds to the region of $0.6<E_{\mathrm{He}^{+}} / E_{\mathrm{O}^{+}}<1$.

The origin of the cross-tail electric field $\varepsilon$ in the Martian magnetotail is quite unclear now. The radius of the Martian magnetotail is just several times larger than the Larmor radius of heavy ion in the plasma sheet and ion kinetic effects in the Martian magnetotail are more important than in the Earth's magnetotail. However, the correlation of heavy ion velocity with the inverse value of the minimum magnetic field in the tail (Kotova et al., 1997, 2000), points on possible existence of the cross-tail electric field in the Martian magnetotail. Estimates done in the paper by Kotova et al. (2000) show that the same formulas for cross-tail electric field acceleration are appropriate for the initial consideration of both magnetotails.

The total energy acquired by a heavy ion after the action of all 3 acceleration mechanisms could be thus estimated:

$$
\frac{E_{i}}{q_{i}}=U+\frac{M_{i} V_{0}^{2}}{2 q_{i}}+\frac{M_{i}(\Delta V)^{2}}{2 q_{i}}+\frac{M_{i} \Delta V}{q_{i}} \sqrt{V_{0}^{2}+\frac{2 q_{i} U}{M_{i}}}
$$

According to the Phobos 2 observations the Martian plasma sheet is primarily filled by oxygen ions and thus it is useful to consider the ratio of the energy per charge gain of an arbitrary ion to the energy of oxygen ion:

$$
E_{\mathrm{O}^{+}}=U+\frac{M_{\mathrm{O}} V_{0}^{2}}{2}+\frac{M_{\mathrm{O}}(\Delta V)^{2}}{2}+M_{\mathrm{O}} \Delta V \sqrt{V_{0}^{2}+\frac{2 U}{M_{\mathrm{O}}}}
$$

$M_{\mathrm{O}}$ is the mass of oxygen ion. It is also convenient to choose the following relative values:

$$
P=\frac{M_{\mathrm{O}} V_{0}^{2}}{2 E_{\mathrm{O}^{+}}}
$$

$$
C=\left(\frac{M_{\mathrm{O}}(\Delta V)^{2}}{2}+M_{\mathrm{O}} \Delta V \sqrt{V_{0}^{2}+\frac{2 U}{M_{\mathrm{O}}}}\right) \cdot \frac{1}{E_{\mathrm{O}^{+}}},
$$

where $P$ is the fraction of the energy gained by oxygen ion due to preacceleration and $C$ is the fraction of the oxygen ion energy acquired in the central current sheet. Obviously the relative input of the field-aligned acceleration $U / E_{\mathrm{O}^{+}}=$ $1-P-C$. Substituting all these parameters into (1), the ratio of the energy per charge gain of an arbitrary ion to the energy of oxygen ion can be expressed as:

$$
\begin{aligned}
\frac{E_{i} / q_{i}}{E_{\mathrm{O}^{+}}}= & {\left[\sqrt{1-P-C+P \frac{M_{i}}{M_{\mathrm{O}} q_{i}}}\right.} \\
& \left.+(1-\sqrt{1-C}) \sqrt{\frac{M_{i}}{M_{\mathrm{O}} q_{i}}}\right]^{2} .
\end{aligned}
$$

The relation (5) determines the ratios $E_{i} / E_{\mathrm{O}^{+}}$for singly charged hydrogen $\left(M_{i}=1\right)$, helium $\left(M_{i}=4\right)$ and molecular oxygen $\left(M_{i}=32\right)$ ions as functions of parameters $P$ and $C$. These parameters determine the relative input of the preacceleration and tail current sheet acceleration, respectively, to the total acceleration of plasma sheet oxygen ions and including the relative input of field-aligned acceleration $U / E_{\mathrm{O}^{+}}$ the sum of the parameters $P, C, U / E_{\mathrm{O}^{+}}$is equal to $100 \%$. Therefore it is convenient to use a liquidus projection to show the results of calculations. Figure 4 presents proper ternary graph of isolines of $E_{\mathrm{H}^{+}} / E_{\mathrm{O}^{+}}, E_{\mathrm{He}^{+}} / E_{\mathrm{O}^{+}}$, and $E_{\mathrm{O}_{2}^{+}} / E_{\mathrm{O}^{+}}$. Every point inside this graph has three coordinates, which are determined in the way shown in the top right corner of Fig. 4. The sum of these coordinates is equal to $100 \%$. Some 
overlapping regions of the parameters $E_{\mathrm{H}^{+}} / E_{\mathrm{O}^{+}}, E_{\mathrm{He}^{+}} / E_{\mathrm{O}^{+}}$, and $E_{\mathrm{O}_{2}^{+}} / E_{\mathrm{O}^{+}}$were selected which agree with the observations summarized in the previous section. These regions are marked by different hatchings. Figure 4 permits to make some quantitative conclusions on the relative influence of the different acceleration processes. It is seen, for example, that when $E_{\mathrm{H}^{+}} / E_{\mathrm{O}^{+}}$is in the range of $0.45-0.7$, then the ratio $E_{\mathrm{O}_{2}^{+}} / E_{\mathrm{O}^{+}}$varies from $\sim 1.2$ to $\sim 1.4$, and $E_{\mathrm{He}^{+}} / E_{\mathrm{O}^{+}}$lies in the range of 0.6-0.8. In this case the relative input of the preacceleration process in the overall plasma sheet ion acceleration may not exceed $40 \%$, the relative input of the magnetic field line stress acceleration and/or field-aligned acceleration is in the range of $30-70 \%$, and the relative input of the cross-tail current sheet acceleration is up to $70 \%$. It is worth to mention that magnetic field line stress acceleration and/or field-aligned electric field acceleration cannot be zero to be consistent with observations from Fig. 4.

Even more strict limitations might be possible. When the relative preacceleration input is $<20 \%$, then bigger difference is allowed between the energies of protons and molecular ions as suggested by observations. This may occur only in the case when the relative input of cross-tail current acceleration is in the interval of $25-55 \%$ and the energy gained by heavy ions due to magnetic field line stress acceleration or electric field aligned acceleration must be in the range of $45-55 \%$. In Fig. 3 the solid and dashed lines illustrate the dependencies $E_{\mathrm{H}^{+}}=0.6 E_{\mathrm{O}^{+}}$and $E_{\mathrm{O}_{2}^{+}}=1.3 E_{\mathrm{O}^{+}}$, respectively, which correspond to the dark point in Fig. 4, where $C=45 \%$ and $P=5 \%$.

It is also possible to check with Eq. (5) whether the energy/charge of $\mathrm{O}_{2}^{+}$relative to the energy of $\mathrm{O}^{+}$corresponds to the observations. For the dark point in Fig. 4 the value of $E_{\mathrm{O}^{+}} / 2 E_{\mathrm{O}^{+}}$is about 0.82 , not contradicting to the observations.

Such a relation between the acceleration processes is also approved by the analysis of the TAUS data, suggesting a preacceleration velocity of at least $45 \mathrm{~km} / \mathrm{s}$ (Kotova et al., 2000 ), while the observed average heavy ion velocity in the Martian plasma sheet was $\sim 140 \mathrm{~km} / \mathrm{s}$ (varied from $\sim 45 \mathrm{~km} / \mathrm{s}$ to $\sim 270 \mathrm{~km} / \mathrm{s}$ ). The relative influence of the cross-tail current sheet and magnetic field line stress acceleration possibly depends on the solar wind ram pressure determining the solar wind interaction with Mars (Kotova et al., 2000; Verigin et al., 1997).

The proposed identification method of relative contribution of possible acceleration processes assumes that the all ion species have undergone the same acceleration processes and took the similar transportation route. This assumption is reasonable for the ion species of planetary origin, while we cannot exclude the possibility that there is a case in which the observed protons came from the solar wind. Since we have rather limited amount of proper data so far this question cannot be resolved now.

\section{Conclusion}

It was demonstrated that the dependence of the energy of heavy ions on their mass could be a useful tool for the identification of possible acceleration processes in the Martian plasma sheet. The study was based on a few cases when heavier ions and protons were registered simultaneously in the plasma sheet by the TAUS energy spectrometer, and on published data of the ASPERA instrument. According to the presented results frictional preacceleration of ions probably in the pole regions of the Martian magnetosphere can be responsible for less than $40 \%$ of the energy gain of heavy ions in the plasma sheet; the input of the cross-tail current sheet acceleration is likely to contribute up to $70 \%$, and from 30 to $70 \%$ of the heavy ion energy is provided by the acceleration due to magnetic field line stresses (and/or electric field aligned acceleration). A further detailed mass analysis of the data may help to confirm this conclusion.

Acknowledgments. The work was supported by OTKA grant T015866 of the Hungarian Science Fund.

\section{References}

Barabash, S. and O. Norberg, Indirect detection of the Martian helium corona, Geophys. Res. Lett., 21, 1547-1550, 1994.

Barabash, S., E. Kallio, R. Lundin, and H. Koskinen, Measurements of the nonthermal helium escape from Mars, J. Geophys. Res., 100, 2130721316, 1995.

Dubinin, E., R. Lundin, O. Norberg, and N. Pissarenko, Ion acceleration in the Martian tail: Phobos observations, J. Geoghys. Res., 98, 3991-3997, 1993.

Eastman, T. E., R. J. DeCoster, and L. A. Frank, Velocity distributions of ion beams in the plasma sheet boundary layer, in Ion Acceleration in the Magnetosphere and Ionosphere, Geophysical Monograph 38, AGU, Washington D.C., pp. 117-126, 1986.

Haerendel, G., Tail exploration and tail formation with artificial plasma clouds, in Magnetotail Physics, edited by A. T. Y. Lui, Johns Hopkins University Press, Baltimore, Md., p. 337, 1987.

Ip, W.-H., Ion acceleration at the current sheet of the Martian magnetosphere, Geophys. Res. Lett., 19, 2095-2098, 1992.

Kotova, G. A., M. I. Verigin, N. M. Shutte, A. P. Remizov, H. Rosenbauer, W. Riedler, K. Schwingnschuh, M. Delva, K. Szegö, and M. Tatrallyay, Planetary heavy ions in the magnetotail of Mars: Results of the TAUS and MAGMA experiments aboard Phobos, Adv. Space Res., 20, 173-176, 1997.

Kotova, G. A., M. I. Verigin, A. P. Remizov, N. M. Shutte, H. Rosenbauer, S. Livi, W. Riedler, K. Schwingenchuh, M. Tatrallyay, K. Szegö, I. Apáthy, Heavy ions in the magnetosphere of Mars: Phobos 2/TAUS observations, Phys. \& Chem. of the Earth (C), 25, 157-160, 2000.

Lichtenegger, H. and E. Dubinin, Model calculations of the planetary ion distribution in the Martian tail, Earth Planets Space, 50, 445-452, 1998

Lundin, R., A. Zakharov, R. Pellinen, H. Borg, B. Hultqvist, N. Pissarenko, E. M. Dubinin, S. W. Barabash, I. Liede, and H. Koskinen, First measurements of the ionospheric plasma escape from Mars, Nature, 341, 609-612, 1989.

Norberg, O., S. Barabash, and R. Lundin, Observations of molecular ions in the Martian plasma environment, in Plasma Environments of Nonmagnetic Planets, COSPAR Colloquia Series, vol. 4, edited by T. Gombosi, p. $299,1993$.

Rosenbauer, H., N. Shutte, I. Apáthy, A. Galeev, K. Gringauz, H. Grünwaldt, P. Hemmerich, K. Jockers, P. Király, G. Kotova, S. Livi, E. Marsch, A. Richter, W. Riedler, A. Remizov, R. Schwenn, K. Schwingenschuh, M. Steller, K. Szegö, M. Verigin, and M. Witte, Ions of Martian origin and plasma sheet in the Martian magnetosphere: initial results of the TAUS experiment, Nature, 341, 612-616, 1989.

Shabanskiy, V. P., Phenomena in the Near Terrestrial Space, pp. 167-190, Nauka, Moscow, 1972 (in Russian).

Speiser, T. W., Particle trajectories in model current sheets, 1. Analitical solutions, J. Geophys. Res., 70, 4219-4226, 1965.

Verigin, M. I., N. Shutte, A. Galeev, K. Gringauz, G. Kotova, A. Remizov, H. Rosenbauer, P. Hemmerich, S. Livi, A. K. Richter, I. Apáthy, K. Szegö, W. Riedler, K. Schwingenschuh, M. Steller, and Ye. G. Yeroshenko, Ions of planetary origin in the Martian magnetosphere (Phobos 2/TAUS experiment), Planet. Space Sci., 39, 131-137, 1991.

Verigin, M., G. Kotova, N. Shutte, A. Remizov, K. Szegö, M. Tatrallyay, I. Apáthy, H. Rosenbauer, S. Livi, A. K. Richter, K. Schwingenschuh, T.-L. Zhang, J. Slavin, and J. Lemaire, Quantitative model of the Martian magnetopause shape and its variation with the solar wind ram pressure based on Phobos 2 observations, J. Geophys. Res., 102, 2147-2155, 1997.

G. A. Kotova (e-mail: kotova@iki.rssi.ru), M. I. Verigin, A. P. Remizov, H. Rosenbauer, S. Livi, W. Riedler, K. Schwingenschuh, M. Tatrallyay, K. Szegö, and I. Apáthy 